\title{
Tradução e adaptação transcultural para a língua portuguesa do Questionário Banff para Instabilidade Patelar*
}

\section{Portuguese Translation and Cross-Cultural Adaption of the Banff Patella Instability Instrument}

\author{
Pedro Henrique Schmidt Alves Ferreira Galvão ${ }^{10}$ Dayane Screpante Marques ${ }^{10}$ \\ Guilherme Conforto Gracitelli ${ }^{10}$ Marcio de Castro Ferreira ${ }^{2(1)}$ Marcelo Seiji Kubota ${ }^{10}$ \\ Carlos Eduardo da Silveira Franciozi ${ }^{1 \oplus}$
}

${ }^{1}$ Departamento de Ortopedia e Traumatologia, Escola Paulista de
Medicina, Universidade Federal de São Paulo, São Paulo, SP, Brasil
${ }^{2}$ Hospital do Coração (HCor), São Paulo, SP, Brazil

Rev Bras Ortop 2021;56(6):747-760.

\author{
Endereço para correspondência Pedro Henrique Schmidt Alves \\ Ferreira Galvão, MD, MSc, Rua Napoleão de Barros, 715, 1 o andar, Vila \\ Clementino, São Paulo, SP, 04024-002, Brasil \\ (e-mail: p.h.galvao63@gmail.com; pgalvao@unifesp.br).
}

\section{Resumo \\ Palavras-chave \\ - luxação patelar \\ - qualidade de vida \\ - inquéritos e questionários \\ - adaptação transcultural \\ - tradução}

Objetivo Realizar a tradução para a língua portuguesa falada no Brasil e a adaptação transcultural do questionário Banff para Instabilidade Patelar.

Métodos A tradução e adaptação transcultural seguiu o processo de validação linguístico proposto por diretrizes internacionais, que consiste em seis etapas: tradução, síntese, retrotradução, revisão pelo comitê de especialistas, pré-teste, e apresentação do relatório final aos autores do questionário original. Foram incluídos no estudo pacientes alfabetizados, com diagnóstico de instabilidade patelar recorrente, com mais de 12 anos de idade, que assinaram ou tiveram o termo de consentimento livre e esclarecido assinado por um responsável legal. Foram excluídos do estudo pacientes portadores de comorbidades neurológicas ou sistêmicas.

Resultados Um total de 62 pacientes ( 18 homens e 44 mulheres) foram incluídos no estudo. Durante os processos de tradução e harmonização das retrotraduções, foram observadas discrepâncias, que foram modificadas sem necessidade de reformulação. Não foram necessárias substituições na versão do pré-teste.

Conclusão O Questionário Banff para Instabilidade Patelar foi traduzido e adaptado transculturalmente para a língua portuguesa falada no Brasil com sucesso, podendo ser
Trabalho realizado pelo Departamento de Ortopedia e Traumatologia, Escola Paulista de Medicina, Universidade Federal de São Paulo, São Paulo, SP, Brasil. recebido

23 de Abril de 2020

aceito

16 de Setembro de 2020

Publicado on-line

Junho 21, 2021
DOI https://doi.org/

10.1055/s-0040-1721840.

ISSN 0102-3616. (c) 2021. Sociedade Brasileira de Ortopedia e Traumatologia. All rights reserved.

This is an open access article published by Thieme under the terms of the Creative Commons Attribution-NonDerivative-NonCommercial-License, permitting copying and reproduction so long as the original work is given appropriate credit. Contents may not be used for commercial purposes, or adapted, remixed, transformed or built upon. (https://creativecommons.org/ licenses/by-nc-nd/4.0/)

Thieme Revinter Publicações Ltda., Rua do Matoso 170, Rio de Janeiro, RJ, CEP 20270-135, Brazil 


\begin{abstract}
utilizado para a avaliação de pacientes portadores de instabilidade patelar falantes desta língua.

Abstract

Keywords
- patellar dislocation
- quality of life
- surveys and
questionnaires
- transcultural
adaptation
- translation

Objective To translate into Brazilian Portuguese and to cross-culturally adapt the Banff Questionnaire for Patellar Instability.

Methods The translation and cross-cultural adaptation followed the linguistic validation process proposed by international guidelines, which consists of six steps: translation, synthesis, back-translation, review by an expert committee, pretest, and final report presentation to the authors of the original questionnaire. Literate patients with recurrent patellar instability, older than 12 years of age, who signed the informed consent form or had it signed by a legal guardian were included in the study. Patients with neurological or systemic comorbidities were excluded from the study.

Results A total of 62 patients ( 18 males and 44 females) were included in the study. Discrepancies observed during the processes of translation and harmonization of the back-translations were modified with no need for reformulation. No pretest version replacements were required.

Conclusion The Banff Questionnaire for Patellar Instability has been successfully translated and cross-culturally adapted into Brazilian Portuguese, so it can be used to assess patients with patellar instability who speak this language.
\end{abstract}

\section{Introdução}

Entre as patologias que acometem o joelho, a instabilidade patelar assume importância não apenas pela incidência com estimativas anuais nos Estados Unidos de 29/100 milhabitantes na população geral, ${ }^{1}$ e até $77 / 100$ mil habitantes em alguns grupos de risco,$-{ }^{2}$ como também pelo fato de ser recorrente em grande parte dos indivíduos acometidos, ${ }^{1}$ atingindo taxas de recorrência de $17 \%$ a $70 \%$ em alguns grupos específicos. ${ }^{3,4}$ Além disso, afeta essencialmente a parcela mais jovem e ativa da sociedade, com pico entre 15 e 19 anos, ${ }^{3}$ o que gera um impacto econômico independente do método de tratamento proposto. ${ }^{5}$

Apesar de comumente ocorrer durante atividades esportivas, ${ }^{4}$ mecanismos atraumáticos foram relatados na presença de condições predisponentes. ${ }^{6}$ A patologia é, por vezes, acompanhada de limitação para atividades recreacionais ou esportivas e da qualidade de vida dos pacientes. ${ }^{7,8}$ Sendo uma condição multifatorial, ${ }^{1-9}$ a instabilidade patelar pode ser manejada com diversas opções terapêuticas, a depender das características anatômicas do paciente e do quadro individualizado. ${ }^{10}$

Ao se utilizar apenas critérios clínicos e radiográficos para a avaliação dos resultados do tratamento proposto, pode-se subestimar o impacto da doença no dia a dia do paciente. $\mathrm{O}$ estado de saúde também deve levar em conta a influência da condição clínica nas diversas situações da vida diária, laboral, recreacional, esportiva e social. ${ }^{11,12}$ Instrumentos de avaliação que abordem a efetividade do tratamento para o paciente e seu impacto na qualidade de vida têm sido elaborados para que se entendam de modo mais amplo os desfechos dos cuidados em saúde. ${ }^{13}$

Questionários como o Kujala ${ }^{14}$ e o International Knee Documentation Committee Subjective Knee Evaluation
Form (IKDC) ${ }^{15}$ são ferramentas já estabelecidas na literatura que apresentam este tipo de aplicação clínica. Ao passo que o IKDC avalia uma grande variedade de patologias do joelho, o Kujala é mais específico para distúrbios da articulação patelofemoral, a fim de documentar especificamente a dor patelofemoral. Ainda assim, as limitações no dia a dia apresentadas por estes pacientes nos aspectos subjetivos podem não ser totalmente compreendidas e dificultar a avaliação das intervenções clínicas. ${ }^{16,17}$

Hiemstra et al. ${ }^{18}$ desenvolveram o questionário Banff para instabilidade patelar, ${ }^{7,18}$ que avalia a qualidade de vida destes pacientes nos aspectos de sintomas e durante a atividade funcional, social e econômica. $\mathrm{O}$ objetivo deste trabalho é traduzir e realizar a adaptação transcultural do questionário Banff para a língua portuguesa falada no Brasil.

\section{Materiais e métodos}

O estudo foi iniciado após aprovação do Comitê de Ética em Pesquisa sob o número CAAE 70103717.3.0000.5505, e a coleta de dados aconteceu no ambulatório do Grupo do Joelho da nossa instituição. Foram incluídos no estudo pacientes alfabetizados, com diagnóstico de instabilidade patelar recorrente, com mais de 12 anos de idade, que assinaram ou tiveram o termo de consentimento livre e esclarecido assinado por um responsável legal. Foram excluídos do estudo pacientes portadores de comorbidades neurológicas ou sistêmicas.

O cálculo amostral foi realizado com base no número de variáveis analisadas, segundo recomendado na literatura, sendo necessário um número mínimo de 62 pacientes, que representa o dobro do número de questões presentes no questionário analisado. ${ }^{19}$ 
Participaram deste estudo 62 pacientes com diagnóstico de instabilidade patelar recorrente. O diagnóstico foi estabelecido quando pelo menos dois episódios de luxação da patela caracterizados na anamnese foram observados pelo paciente ou por terceiros, associados ao exame clínico e a métodos de imagem, como descrito por Brattstroem. ${ }^{20}$

A tradução e adaptação transcultural do Questionário Banff para Instabilidade Patelar para a língua portuguesa falada no Brasil seguiu o processo de validação linguística internacionalmente aceito descrito por Guillemin et al. ${ }^{21} \mathrm{e}$ adaptado por Beaton et al. ${ }^{22,23} \mathrm{O}$ objetivo de se utilizar um processo de validação linguística é obter uma tradução que tenha equivalência à original e que também possa ser compreendida pela população-alvo. O método utilizado neste estudo é descrito a seguir (-Figura 1).

1. Tradução: iniciou-se após a obtenção da autorização para o uso do questionário pelos autores do artigo original, a revisão dos itens do questionário, e a organização do material e do fluxo de coleta de dados para o desenvolvimento do trabalho - processo de "Preparação". As 32 questões, instruções, opções de resposta, e demais itens do questionário original em inglês foram traduzidas para o português, de maneira independente, por 2 tradutores cirurgiões ortopedistas brasileiros fluentes em ambas as línguas, dando origem a 2 versões traduzidas (Banff VT1 e Banff VT2).

2. Síntese: ambas as versões geradas foram comparadas em uma reunião com um comitê de especialistas, a partir da qual se originou uma "Banff Versão T12” (Banff VT12).

3. Retrotradução: esta última Banff VT12 foi retrotraduzida ("back-translated") por outros dois tradutores, estes de língua-mãe inglesa, com fluência em português, cegados quanto ao questionário original e sem conhecimento sobre o assunto. Entre os objetivos desta etapa estiveram encontrar erros conceituais de tradução e inconsistências grosseiras das fases anteriores. Geraram-se, então, duas "versões retrotraduzidas" (Banff VRT1 e Banff VRT2).

4. Revisão pelo comitê de especialistas: foi realizada uma nova reunião, agora entre o terceiro e quarto tradutores e o mesmo comitê, para buscar inconsistências e verificar a correspondência entre as versões retrotraduzidas (Banff VRT1 e Banff VRT2), a tradução inicial (Banff VT12), e o questionário original. A harmonização das discrepâncias, buscando a equivalência semântica, idiomática, experiencial e conceitual entre as versões, gerou a "Banff Versão Brasileira Pré-Final" (Banff VBPF) em português. Esta versão teve o papel de consolidar todas as informações produzidas até então em um instrumento de fácil compreensão que foi utilizado para o pré-teste com a amostra populacional inclusa no estudo.

5. Pré-teste: foi conduzido no ambulatório do Grupo do Joelho do Hospital São Paulo, no Departamento de Ortopedia e Traumatologia da Escola Paulista de Medicina, Universidade Federal de São Paulo (UNIFESP), sendo aplicada a Banff VBPF pela técnica de sondagem: ${ }^{21}$ após a aplicação do questionário, sondou-se individualmente cada paciente quanto à clareza, compreensão e aceitabilidade de cada um dos itens presentes no instrumento. No caso de dúvidas ou sugestões, poderia haver a necessidade de reformular algum item, sendo isto discutido entre o comitê. Caso contrário, proceder-se-ia à última etapa.

6. Apresentação da versão final aos autores do questionário original: foi enviada a documentação referente aos relatórios elaborados ao longo dos demais estágios, assim como quais as discrepâncias que surgiram e qual foi o consenso do comitê para a formulação de cada item da Banff VBPF. A autora principal do questionário original aprovou a versão encaminhada sem sugestões ou alterações. Esta versão passou a ser designada "Banff Versão Brasileira Final" (Banff VBF).

\section{Resultados}

O pré-teste ocorreu de junho de 2018 até agosto de 2019, no ambulatório do Grupo do Joelho, no Departamento de Ortopedia e Traumatologia. Quanto à caracterização da amostra populacional, o estudo incluiu um total de 62 pacientes. A idade média correspondeu a 29,2 anos (desvio padrão = 11,6), com mínima de 12 anos e valor máximo de 57 anos. Houve um predomínio de pacientes do sexo feminino: 44 (70,96\%), enquanto 18 (29\%) eram do sexo masculino. Os valores do escore Banff estão resumidos na - Tabela 1.

Durante a reconciliação das versões VT1 e VT2, o comitê de especialistas encontrou um total de 22 itens com discrepâncias nas 32 perguntas, 19 itens com discrepâncias nas 32 respostas, e 18 itens discrepantes nas 21 frases de informação, título, ou instruções presentes no questionário original. Salientando-se a necessidade de manter a equivalência, conforme proposto por Guillemin et al., ${ }^{21}$ após a análise dos itens individualmente pelos membros do comitê, buscou-se obter um consenso na síntese entre as traduções, na tentativa de manter as propriedades da versão original.

O questionário VT12, submetido à retrotradução, resultou em duas versões em inglês, conforme mostra a - Figura 1: VRT1 e VRT2. Durante a sua harmonização, a equivalência transcultural $^{22}$ semântica, idiomática, experiencial e conceitual de cada item em relação à sua versão original foi analisada sem necessidade de reformulação pelos 5 membros do comitê quando o índice de concordância encontrado foi acima de $80 \%{ }^{24}$

Foi necessária apenas uma análise pelo comitê de especialistas. Verificando as versões retrotraduzidas, identificaram-se 26 itens com discrepâncias entre as 32 questões, 29 itens com discrepâncias nas 32 respostas, e 20 discrepâncias nas 21 frases de informação, título ou instrução. Com a resolução de todas as discrepâncias encontradas, formulou-se a VBPF. $O$ comitê pontuou a necessidade de algumas adaptações para a elaboração da VBPF, em busca de manter a equivalência à versão original, permitindo ao mesmo tempo que este instrumento fosse compreensível para a população brasileira, sem alterar as medidas analisadas. $\mathrm{O}$ resumo deste processo está exemplificado na - Tabela 2.

Durante o curso da validação linguística, não foram registrados no pré-teste questões, respostas ou outros itens não compreendidos pelos pacientes avaliados. Portanto, a versão pré-final do questionário foi enviada, sem necessidade de reformulações, aos autores do questionário original, 


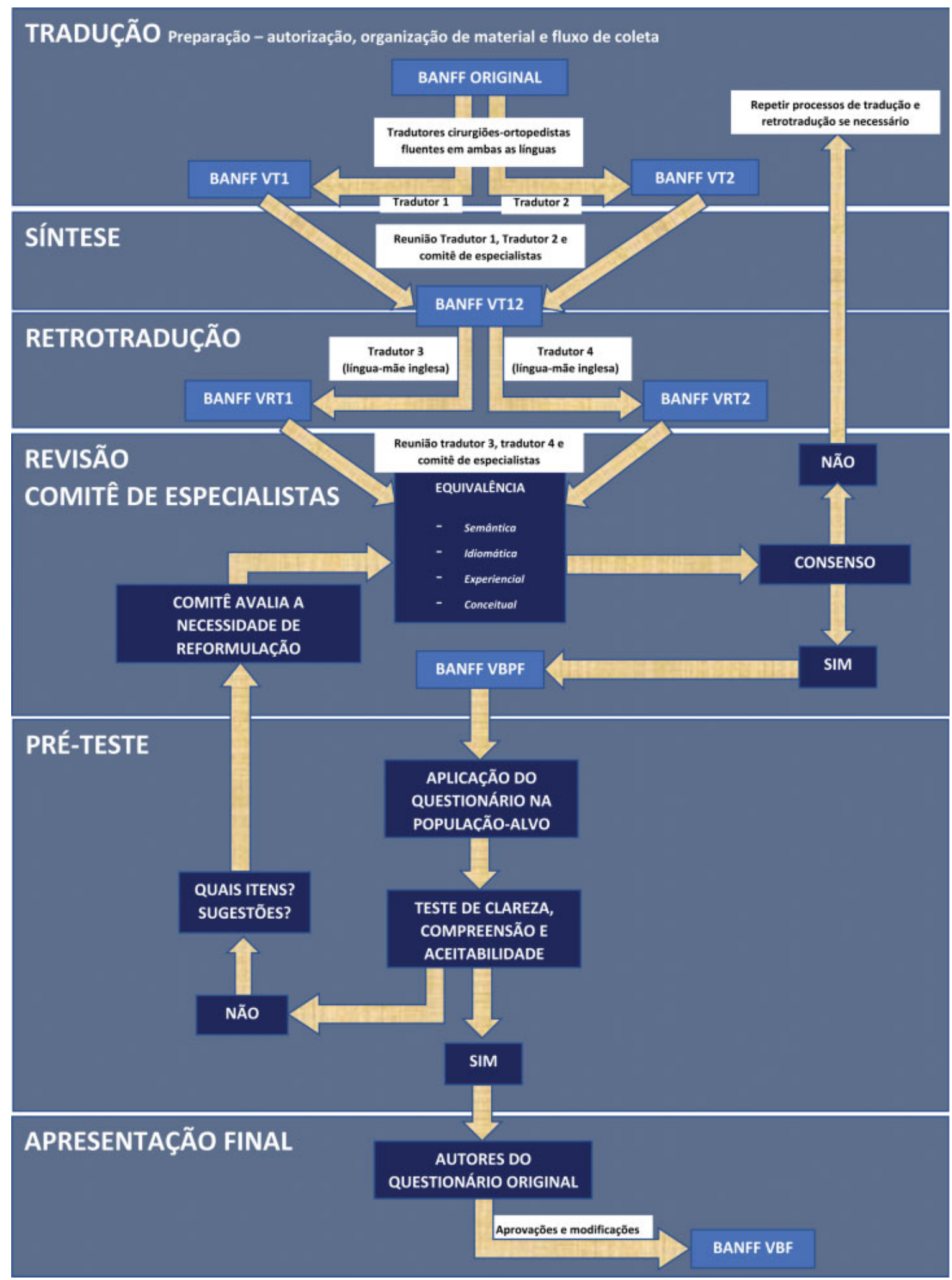

Fig. 1 Fluxograma de validação linguística do Questionário Banff Versão Brasileira. Abreviaturas: VT1, versão traduzida 1; VT2, versão traduzida 2; VT12, versão traduzida 12; VRT1, versão retrotraduzida 1; VRT2, versão retrotraduzida 2; VBPF, versão brasileira pré-final; VBF, versão brasileira final.

juntamente do relatório das adaptações realizadas ao longo do processo. Em correspondência com a autora do questionário original, esta versão foi aprovada, sem qualquer modi- ficação sugerida, como a versão brasileira final (VBF), que passou a ser denominada Questionário Banff para Instabilidade Patelar Versão Brasileira, disponível no Apêndice 1. 
Tabela 1 Escore de Banff Pré-Teste

\begin{tabular}{|l|l|l|l|l|l|}
\hline Escore de Banff & Médio & Desvio padrão & Mínimo & Máximo & IC95\% \\
\hline Geral & 30,33 & 15,7 & 4,68 & 86,5 & $26,71-33,96$ \\
\hline Sexo masculino & 33,09 & 19,04 & & & $24,65-41,54$ \\
\hline Sexo feminino & 29,19 & 14,21 & & & $25,27-33,11$ \\
\hline
\end{tabular}

Abreviatura: IC, Intervalo de confiança.

Tabela 2 Exemplos de itens do Banff Patella Instability Instrument submetidos à adaptação transcultural

\begin{tabular}{|c|c|c|c|c|}
\hline Questão & Versão original (BPII) & Tradução (VT12) & Alterações para VBPF & Comentário \\
\hline $1 a$ & $\begin{array}{l}\text { "... severity of the 'giv- } \\
\text { ing way' episodes?" }\end{array}$ & $\begin{array}{l}\text { “... episódios em que a } \\
\text { rótula (patela) sai do } \\
\text { lugar? (Gravidade)" }\end{array}$ & $\begin{array}{l}\text { “... episódios em que a } \\
\text { rótula sai do lugar? Qual } \\
\text { a gravidade dos episó- } \\
\text { dios de deslocamento?" }\end{array}$ & $\begin{array}{l}\text { Expressão sem equivalência idio- } \\
\text { mática no português. Para manter } \\
\text { compreensível à população brasi- } \\
\text { leira, preservando sua equivalên- } \\
\text { cia conceitual, foi evitado o termo } \\
\text { "luxação", e utilizado "rótula sai do } \\
\text { lugar" e "deslocamento" }\end{array}$ \\
\hline 3 & “... stiffness” & “... joelho duro” & “... joelho duro” & $\begin{array}{l}\text { Embora o comitê tivesse julgado que } \\
\text { a equivalência semântica mais } \\
\text { adequada fosse "rigidez", a expres- } \\
\text { são “joelho duro" manteve a mesma } \\
\text { equivalência conceitual, com uma } \\
\text { maior compreensão. Assim, optou- } \\
\text { se por mantê-la na VBPF }\end{array}$ \\
\hline 9 & $\begin{array}{l}\text { “... sudden twisting and } \\
\text { pivoting movements or } \\
\text { changes in direction" }\end{array}$ & $\begin{array}{l}\text { “... movimentos de } \\
\text { giro/rotação repentinos } \\
\text { ou movimentos de } \\
\text { mudanças de direção } \\
\text { repentinos" }\end{array}$ & $\begin{array}{l}\text { “... movimentos rota- } \\
\text { cionais ou de mudanças } \\
\text { bruscas de direção" }\end{array}$ & $\begin{array}{l}\text { Tentativa de simplificar os termos } \\
\text { elaborados na tradução (VT12), } \\
\text { mantendo sua equivalência } \\
\text { conceitual }\end{array}$ \\
\hline 14 & “... knee 'giving way'” & $\begin{array}{l}\text { “... joelho ‘sair do } \\
\text { lugar'” }\end{array}$ & $\begin{array}{l}\text { “.. rótula (patela) sair } \\
\text { do lugar” }\end{array}$ & $\begin{array}{l}\text { Expressão sem equivalência idio- } \\
\text { mática no português. Substituído } \\
\text { “joelho" da tradução (VT12) por } \\
\text { "rótula (patela)", pois, de acordo } \\
\text { com consenso do comitê, o termo } \\
\text { na tradução sugeria a ideia de todo } \\
\text { o joelho se deslocar, em vez de a } \\
\text { patela em si }\end{array}$ \\
\hline 28 & $\begin{array}{l}\text { “... to psychologically } \\
\text { "come to grips'?” }\end{array}$ & $\begin{array}{l}\text { “... superar } \\
\text { psicologicamente” }\end{array}$ & $\begin{array}{l}\text { “... superar } \\
\text { psicologicamente” }\end{array}$ & $\begin{array}{l}\text { Expressão sem equivalência idio- } \\
\text { mática no português. Traduzida } \\
\text { por expressão de equivalência } \\
\text { conceitual similar. Optou-se por } \\
\text { mantê-la na VBPF }\end{array}$ \\
\hline
\end{tabular}

Abreviaturas: BPII, Banff Patella Instability Instrument; VT12, versão traduzida 12; VBPF, versão brasileira pré-final.

\section{Discussão}

Identifica-se na literatura uma preocupação cada vez maior, não só na melhora da satisfação relatada pelos pacientes, como também no desenvolvimento de medidas de desfecho para uma população ou uma condição clínica específica. ${ }^{25}$ Sendo a instabilidade patelar uma patologia multifatorial, ${ }^{1-10,26-28}$ é necessário que se utilizem ferramentas adequadas que permitam a comparação entre diferentes estratégias de tratamento. Um resumo dos instrumentos utilizados na avaliação da instabilidade patelar relatado por Hiemstra et al. ${ }^{25}$ é encontrado na - Figura 2.

Constata-se, a partir destes dados, que alguns questionários que foram utilizados durante muitos anos para a avaliação de distúrbios da articulação patelofemoral têm enfoque em caracterizar outras patologias do joelho, atribuindo muitas vezes pesos elevados a itens que não estão necessariamente presentes neste tipo de lesão. O escore de Lysholm, por exemplo, traduzido e validado para o uso na língua portuguesa, ${ }^{29}$ tem um total de 60 dos seus 100 pontos mensurando dor e instabilidade, o que o torna inadequado para estimar dor anterior. ${ }^{14}$ Já o questionário Kujala "scoring of patellofemoral disorders", que também se apresenta em uma versão traduzida e adaptada culturalmente para o português, ${ }^{30}$ embora amplamente utilizado para avaliar pacientes com histórico prévio de luxação da patela, tem apenas 1 de suas 13 questões direcionadas especificamente para a instabilidade patelar.

O Banff Patella Instability Instrument (BPII) foi originalmente publicado em 2013 no Canadá por Hiemstra et al., ${ }^{18}$ na tentativa de preencher esta lacuna então relatada à época, ${ }^{31} \mathrm{da}$ 


\begin{tabular}{|c|c|c|c|c|c|c|c|}
\hline Instrumentos ${ }^{*}$ & Lysholm & Kujala & $\begin{array}{l}\text { IKDC } \\
\text { modificado }\end{array}$ & Pedi-IKDC & Koos & Koos-Child & NPI \\
\hline Ano de publicaçâo & 1982 & 1993 & 2001 & 2011 & 1998 & 2012 & 2012 \\
\hline Construto avaliado & $\begin{array}{l}\text { Instabilidade } \\
\text { ligamentar }\end{array}$ & $\begin{array}{c}\text { Dor anterior } \\
\text { no joelho }\end{array}$ & $\begin{array}{l}\text { Lesōes no } \\
\text { joelho }\end{array}$ & $\begin{array}{l}\text { Lesōes no } \\
\text { joelho }\end{array}$ & $\begin{array}{l}\text { Lesōes no joelho; } \\
\text { inclui osteoartrite }\end{array}$ & Lesōes no joelho & $\begin{array}{c}\text { Instabilidade } \\
\text { patelar }\end{array}$ \\
\hline População-alvo & $\begin{array}{c}\text { Acima de } 12 \\
\text { anos e adultos }\end{array}$ & Adultos & Adultos & $10-18$ anos & $\begin{array}{c}16 \text { anos, adultos } \\
\text { e idosos }\end{array}$ & $7-18$ anos & Adultos \\
\hline Número de itens & 8 & 13 & 18 & 13 & 42 & 39 & 19 \\
\hline Modelo de resposta & $\begin{array}{l}\text { Múltipla } \\
\text { escolha }\end{array}$ & $\begin{array}{l}\text { Múltipla } \\
\text { escolha }\end{array}$ & Escala Likert & Escala Likert & Escala Likert & Escala Likert & Escala Likert \\
\hline Pontuaçäo & $\begin{array}{l}\text { Variável de } \\
\text { acordo com o } \\
\text { item medido }\end{array}$ & $\begin{array}{c}\text { Variável de } \\
\text { acordo com } \\
\text { o item } \\
\text { medido }\end{array}$ & $\begin{array}{c}\text { Itens com } \\
\text { peso } \\
\text { estabelecido }\end{array}$ & $\begin{array}{c}\text { Itens com } \\
\text { peso } \\
\text { estabelecido }\end{array}$ & $\begin{array}{c}\text { Dominios } \\
\text { avaliados } \\
\text { separadamente }\end{array}$ & $\begin{array}{l}\text { Itens com peso } \\
\text { estabelecido; } \\
\text { dominios avaliados } \\
\text { separadamente }\end{array}$ & $\begin{array}{c}\text { Itens com } \\
\text { peso } \\
\text { estabelecido }\end{array}$ \\
\hline Faixa de pontuaçăo & $0-100$ & $0-100$ & $0-100$ & $0-100$ & $\begin{array}{l}\text { 0-100 para cada } \\
\text { domínio }\end{array}$ & $0-100$ & $0-100$ \\
\hline Tempo para completar & $3 \mathrm{~min}$ & $4 \mathrm{~min}$ & $10 \mathrm{~min}$ & $10 \mathrm{~min}$ & $10 \mathrm{~min}$ & $10-20 \mathrm{~min}$ & $4 \mathrm{~min}$ \\
\hline Idioma original & Sueco e inglês & $\begin{array}{c}\text { Finlandès e } \\
\text { inglês }\end{array}$ & Inglês & Inglês & Inglês e sueco & Inglês e sueco & Inglès \\
\hline Traduçes disponiveis & $>5$ & $>10$ & $>10$ & Dinamarquês & $>50$ & $>5$ & Alemăo \\
\hline
\end{tabular}

Fig. 2 Quadro dos instrumentos utilizados na avaliação de distúrbios patelofemorais, exceto Banff. Abreviaturas: IKDC, International Knee Documentation Committee; KOOS, Knee Injury and Osteoarthritis Outcome Score; NPI, Norwich Patellar Instability.

falta de um questionário específico que avaliasse a instabilidade patelar nos modelos de "Patient-Reported Outcome Measures". As 32 questões pertencentes a 5 domínios distintos foram elencadas por um procedimento de Ebel modificado realizado por um grupo internacional de especialistas -, a fim de se identificar quais as medidas de desfecho específicas eram mais relevantes no contexto da instabilidade patelar. ${ }^{7}$

Sendo o peso atribuído a cada resposta semelhante entre os diferentes itens, o escore final consiste na média de todas as respostas nos cinco domínios abordados, com um resultado final mais alto refletindo uma melhor qualidade de vida. ${ }^{25}$ Este método fornece ao questionário Banff a possibilidade de avaliar, por meio de uma visão mais abrangente, a qualidade de vida dos pacientes com instabilidade patelar. ${ }^{7}$

Embora o Norwich Patella Instability Score ${ }^{32}$ tenha surgido da mesma demanda e contexto que o BPII, de analisar medidas de desfecho de pacientes com instabilidade patelar, seu enfoque é na caracterização dos sintomas físicos gerados pelo quadro clínico. O peso atribuído aos seus 19 itens obedece um algoritmo complexo: itens que elencam atividades que mais comumente gerariam sintomas de instabilidade recebem uma pontuação máxima de menor valor; já aquelas atividades que não gerariam estes mesmos sintomas para a maior parte dos pacientes, exceto em instabilidades mais acentuadas, têm o potencial de receber mais pontos. Deste modo, um escore final elevado indica maiores graus de instabilidade, e, portanto, pior função.

Identificando os questionários sobre instabilidade patelar disponíveis na literatura, a minoria dos instrumentos de mensuração foi submetida à validação entre as nove esferas possíveis, conforme as recomendações da Consensus-based Standards for the Selection of Health Status Measurement Instruments (COSMIN). ${ }^{33,34}$ O BPII já passou pela análise de várias propriedades psicométricas, incluindo validade de conteúdo, consistência interna e confiabilidade, ${ }^{18}$ validade de construto, validade de critério, ${ }^{7}$ entre outras.
Em 2016, o BPII passou por uma análise de fatores e redução de itens, ${ }^{34}$ sendo reduzido ao BPII 2.0, que contém 23 itens, ainda divididos nos mesmos 5 domínios de sua primeira versão. Esta redução se deu em parte pelo fato de muitos pacientes não responderem à totalidade das questões, tentando-se adequar as questões também à população pediátrica; além disso, foi observado, com o BPII 2.0, um número menor de questões não respondidas, conforme relatado pela autora do questionário original em correspondência por e-mail.

Diversas propriedades psicométricas do BPII 2.0 foram testadas e somaram ao processo de validação deste instrumento. Entre elas, pode-se citar um estudo multicêntrico que evidenciou a validação do BPII 2.0 ao Pedi-IKDC, ${ }^{35} \mathrm{com}$ correlação moderada, assim como a validação transcultural para o alemão, que incluiu as populações alemã, austríaca, e suíça. ${ }^{36}$ Além disso, encontra-se em processo de validação para o holandês, espanhol, finlandês e francês. ${ }^{25}$

Mesmo após estabelecido um consenso, algumas perguntas da versão final da tradução para o português geraram dúvidas no comitê quanto à aceitabilidade da população-alvo de certas construções gramaticais (exemplo: "quanto medo"). Ainda assim, todas as questões foram compreendidas pela totalidade dos participantes durante o pré-teste, sem sugestões de modificações. Outra limitação deste estudo foi realizar apenas a tradução e a adaptação transcultural do Questionário Banff para Instabilidade Patelar. A validação, por sua vez, é um processo complexo e iterativo, de modo que estudos adicionais serão necessários para aumentar a amostra representativa da população brasileira. Esta etapa se encontra em andamento pelo presente grupo deste estudo.

\section{Conclusão}

O BPII foi submetido à tradução e adaptação transcultural para a língua portuguesa falada no Brasil com sucesso, e pode 
ser aplicado na avaliação da qualidade de vida dos pacientes com instabilidade patelar no Brasil.

\section{Suporte Financeiro}

Não houve suporte financeiro de fontes públicas, comerciais, ou sem fins lucrativos.

\section{Conflito de Interesses}

Os autores declaram não haver conflito de interesses. $\mathrm{O}$ dr. Galvao informa que fez um pedido de patente para o Questionário Banff para Instabilidade Patelar Versão Brasileira.

\section{Referências}

1 Colvin AC, West RV. Patellar instability. J Bone Joint Surg Am 2008; 90(12):2751-2762

2 Sanders TL, Pareek A, Hewett TE, Stuart MJ, Dahm DL, Krych AJ. Incidence of First-Time Lateral Patellar Dislocation: A 21-Year Population-Based Study. Sports Health 2018;10(02):146-151

3 Waterman BR, Belmont PJ Jr, Owens BD. Patellar dislocation in the United States: role of sex, age, race, and athletic participation. J Knee Surg 2012;25(01):51-57

4 Fithian DC, Paxton EW, Stone ML, et al. Epidemiology and natural history of acute patellar dislocation. Am J Sports Med 2004;32 (05):1114-1121

5 Nwachukwu BU, So C, Schairer WW, et al. Economic Decision Model for First-Time Traumatic Patellar Dislocations in Adolescents. Am J Sports Med 2017;45(10):2267-2275

6 Aglietti P. Disorders of the patellofemoral joint. In: Insall JN, Windsor RE, Scott WN, Kelly MA, Aglietti P, editors. Surgery of the Knee. Philadelphia: Churchill Livingstone; 2001:913-1045

7 Hiemstra LA, Kerslake S, Lafave M, Mohtadi NG. Concurrent Validation of the Banff Patella Instability Instrument to the Norwich Patellar Instability Score and the Kujala Score in Patients With Patellofemoral Instability. Orthop J Sports Med 2016;4(05): 2325967116646085

8 Hiemstra LA, Kerslake S, Lafave M. Medial Patellofemoral Ligament Reconstruction Femoral Tunnel Accuracy: Relationship to Disease-Specific Quality of Life. Orthop J Sports Med 2017;5(02): 2325967116687749

9 Petri M, Ettinger M, Stuebig T, et al. Current Concepts for Patellar Dislocation. Arch Trauma Res 2015;4(03):e29301

10 Kader D, Matar H, Caplan N. Patellofemoral joint instability: a review of current concepts. J Orthop Trauma 2016;6(01):1-8

11 Berlim MT, Fleck MP. Qualidade de vida: um novo conceito para a pesquisa e prática em psiquiatria. Rev Bras Psiquiatr 2003;25(04): 249-252

12 Duarte PS, Miyazaki MC, Ciconelli RM, Sesso R. Tradução e adaptação cultural do instrumento de avaliação de qualidade de vida para pacientes renais crônicos (KDQOL-SF). Rev Assoc Med Bras (1992) 2003;49(04):375-381

13 Lopes AD, Stadniky SP, Masiero D, Carrera EF, Ciconelli RM, Griffin S. Tradução e adaptação cultural do WORC: um questionário de qualidade de vida para alterações do manguito rotador. Rev Bras Fisioter São Carlos 2006;10(03):309-315

14 Kujala UM, Jaakkola LH, Koskinen SK, Taimela S, Hurme M, Nelimarkka O. Scoring of patellofemoral disorders. Arthroscopy 1993;9(02):159-163

15 Hefti F, Müller W, Jakob RP, Stäubli HU. Evaluation of knee ligament injuries with the IKDC form. Knee Surg Sports Traumatol Arthrosc 1993;1(3-4):226-234

16 Acquadro C, Conway K, Girourdet C, Mear I. Linguistic Validation Manual for Patient Reported Outcomes(PRO) Instruments. Lyon
(France)MAPI Research Trust2004. Available from: URL: http:// www.mapi-research.fr/i_02_manu.htm

17 Acquadro C, Janbom B, Ellis D, Marquis P. Language and translation issues. In: Clinical trials. 2nd ed. Philadelphia: LippincottRaven; 1996:575-585

18 Hiemstra LA, Kerslake S, Lafave MR, Heard SM, Buchko GM, Mohtadi NG. Initial validity and reliability of the Banff Patella Instability Instrument. Am J Sports Med 2013;41(07):1629-1635

19 Hair JF, Black B, Babin B, Anderson RE, Tatham RL. Multivariate Data Analysis. 6th ed. Bill Black, Louisiana State University; 2006

20 Brattstroem $\mathrm{H}$. Shape of the intercondylar groove normally and in recurrent dislocation of patella. a clinical and x-ray-anatomical investigation. Acta Orthop Scand Suppl 1964;68(Suppl 68):68, 1-148

21 Guillemin F, Bombardier C, Beaton D. Cross-cultural adaptation of health-related quality of life measures: literature review and proposed guidelines. J Clin Epidemiol 1993;46(12):1417-1432

22 Beaton DE, Bombardier C, Guillemin F, Ferraz MB. Guidelines for the process of cross-cultural adaptation of self-report measures. Spine 2000;25(24):3186-3191

23 Beaton D, Bombardier C, Guillemin F, Ferraz M. Recommendations for the cross-cultural adaptation of the DASH \& QuickDASH outcome measures. Institute Work Health 2007;1(01):1-45

24 Pasquali L. Princípios de elaboração de escalas psicológicas. Rev Psiquiatr Clin (Santiago) 1998;25(05):206-213

25 Hiemstra LA, Page JL, Kerslake S. Patient-reported outcome measures for patellofemoral instability: a critical review. Curr Rev Musculoskelet Med 2019;12(02):124-137

26 Franciozi CE, Ambra LF, Albertoni LJB, et al. Anteromedial Tibial Tubercle Osteotomy Improves Results of Medial Patellofemoral Ligament Reconstruction for Recurrent Patellar Instability in Patients With Tibial Tuberosity-Trochlear Groove Distance of 17 to $20 \mathrm{~mm}$. Arthroscopy 2019;35(02):566-574

27 Franciozi CE, Ambra LF, Albertoni LJ, et al. Increased Femoral Anteversion Influence Over Surgically Treated Recurrent Patellar Instability Patients. Arthroscopy 2017;33(03):633-640

28 Gobbi RG, Demange MK, de Ávila LFR, et al. Patellar tracking after isolated medial patellofemoral ligament reconstruction: dynamic evaluation using computed tomography. Knee Surg Sports Traumatol Arthrosc 2017;25(10):3197-3205

29 Peccin MS, Ciconelli R, Cohen M. Questionário específico para sintomas do joelho "Lysholm Knee Scoring Scale": tradução e validação para a língua portuguesa. Acta Ortop Bras 2006;14(05):268-272

30 Aquino VS, Falcon SFM, Neves LMT, Rodrigues RC, Sendín FA. Tradução e adaptação cultural para a língua portuguesa do questionário scoring of patellofemoral disorders: estudo preliminar. Acta Ortop Bras 2011;19(05):273-279

31 Smith TO, Davies L, O'Driscoll ML, Donell ST. An evaluation of the clinical tests and outcome measures used to assess patellar instability. Knee 2008;15(04):255-262

32 Smith TO, Donell ST, Clark A, et al. The development, validation and internal consistency of the Norwich Patellar Instability (NPI) score. Knee Surg Sports Traumatol Arthrosc 2014;22(02):324-335

33 Mokkink LB, Terwee CB, Patrick DL, et al. The COSMIN checklist for assessing the methodological quality of studies on measurement properties of health status measurement instruments: an international Delphi study. Qual Life Res 2010;19(04):539-549

34 Lafave MR, Hiemstra L, Kerslake S. Factor Analysis and Item Reduction of the Banff Patella Instability Instrument (BPII): Introduction of BPII 2.0. Am J Sports Med 2016;44(08):2081-2086

35 Lafave MR, Hiemstra LA, Parikh SN, Peterson D, Kerslake S. Validity and Reliability of the Banff Patellofemoral Instability Instrument 2.0 in an Adolescent Population. J Pediatr Orthop 2020;40(02):e103-e108

36 Becher C, Attal R, Balcarek P, et al. Successful adaption of the Banff Patella Instability Instrument (BPII) 2.0 into German. Knee Surg Sports Traumatol Arthrosc 2018;26(09):2679-2684 


\section{QUESTIONÁRIO BANFF PARA INSTABILIDADE PATELAR VERSÃO BRASILEIRA}

Escore de qualidade de vida para pacientes com instabilidade patelar

Nome do paciente (nome completo)

Data (dia/mês/ano): Sexo:

Telefone para contato: E-mail para contato

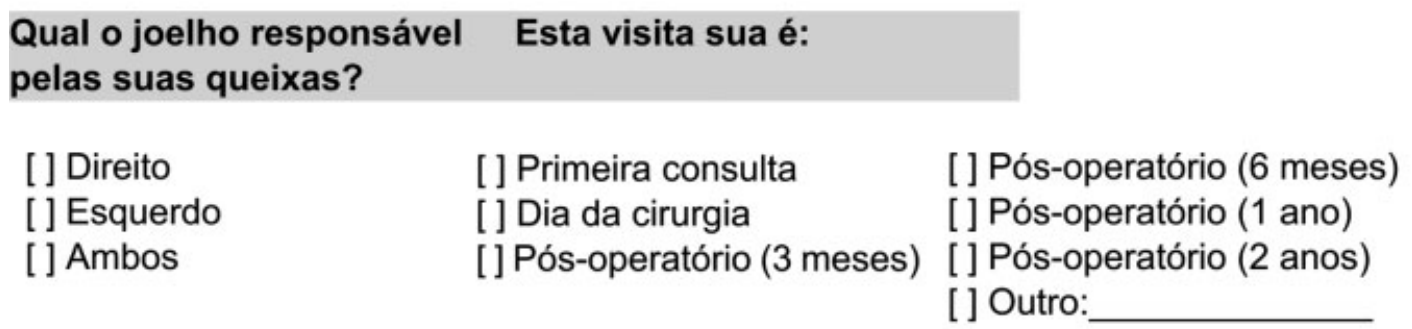

\section{INSTRUÇÕES}

Responda as questões abaixo considerando os últimos três meses, a respeito do estado atual, função, atividades do dia-a-dia e sensação de insegurança que o seu joelho proporciona por conta da instabilidade patelar (rótula que sai do lugar). Para cada uma das questões abaixo haverá uma linha contínua de 0 a 100. Por favor, indique com um "risco" ("l") sobre o ponto que mais se aproxima de sua resposta para cada uma das situações solicitadas.

Este é um exemplo:

\section{Este é um bom questionário?}

$$
0
$$
100

Inútil

\section{Excelente}

Caso você posicione o "/" no meio da linha, isto indica que o questionário é de qualidade moderada, pois a resposta se encontra no meio do caminho entre "Inútil" e "Excelente". Já se a sua resposta representar o extremo de cada situação, é importante você posicionar o "/"no início ("Inútil") ou no final da linha ("Excelente"), refletindo a sua condição.

Apêndice 1 Questionário Banff para Instabilidade Patelar Versão Brasileira. 


\section{SEÇÃO A: SINTOMAS E QUEIXAS FÍSICAS}

As primeiras quatro questões estão relacionadas a SINTOMAS E QUEIXAS FísICAS

1) A respeito da função geral do seu joelho, quão problemáticos são os episódios em que a rótula sai do lugar?

Marque o "/" à direita do final da linha caso você não esteja apresentando nenhum episódio de deslocamento da rótula neste período. Esta pergunta tem duas partes: (1.a) e (1.b)

1.a) Qual a gravidade dos episódios de deslocamento?

0

$$
\text { Episódios }
$$

muito graves
100

Episódios

pouco graves

1.b) Qual a frequência dos episódios de deslocamento?

$\begin{array}{cr}0 & 100 \\ \text { Sempre } & \text { Nunca } \\ \text { acontecem } & \text { acontecem }\end{array}$

2) Quanto de desconforto ou dor você sente no joelho durante uma atividade prolongada (por exemplo, acima de 30 minutos)?

$$
0
$$
100

$$
\text { Dor }
$$

Sem

intensa

dor

3) Sobre a função do seu joelho envolvido, quanto você se incomoda com a perda de movimento do joelho ou com ficar com o "joelho duro"?

$\begin{array}{cc}\text { Muito } & 100 \\ \text { incomodado } & \text { Nada } \\ \text { incomodado }\end{array}$

4) Considerando a função do seu joelho e sua relação com a força muscular: quão fraco é o seu joelho?

$$
0
$$
100

\section{Muito \\ fraco}

Sem

fraqueza 


\section{SEÇÃO B: QUEIXAS RELACIONADAS AO TRABALHO}

As questões abaixo se relacionam à sua profissão ou vocação e se referem à sua funcionalidade no trabalho e qualquer queixa relacionada ao trabalho. Caso você seja um estudante em tempo integral ou trabalhe no seu domicílio, considere esta atividade como a sua profissão. Também pode ser considerado nesta seção qualquer trabalho temporário extra. Leve em conta os últimos três meses para responder as perguntas abaixo.

Se você é DESEMPREGADO por outras razões que NÃO RELACIONADAS AO SEU JOELHO, marque um " $X$ " nesta linha:

5) Quanto de dificuldade você encontra, por conta do seu joelho, para realizar movimentos de mudança de direção ou de rotação do corpo sobre o joelho? (Marque o "/" no início da linha à esquerda se você se encontra incapaz de trabalhar devido ao seu joelho)

0 100

Completamente incapaz
Não me gera

limitações

6) Quanto de dificuldade você encontra, por conta do seu joelho, para realizar movimentos de agachamento no seu trabalho? (Marque o "/" no início da linha à esquerda se você se encontra incapaz de trabalhar devido ao seu joelho)

0 100

\section{Muita}

Sem

dificuldade

dificuldade

7) Quanto você se preocupa em perder dias de trabalho devido a problemas ou novas lesões no joelho? (Marque o "/" no início da linha à esquerda se você se encontra incapaz de trabalhar devido ao seu joelho)

$$
0
$$
100

É um problema

Não me

extremamente

preocupa

significante

8) Quanto você se preocupa em perder aulas no colégio, faculdade ou ainda tempo de trabalho devido ao seu tratamento do joelho?

0 100

\section{É um problema}

Não me

extremamente

preocupa

\section{significante}

Apêndice 1 (continued). 


\section{SEÇÃO C: ESPORTES/ATIVIDADES RECREACIONAIS/COMPETITIVAS} As questões abaixo se referem a ATIVIDADES RECREACIONAIS E PARTICIPAÇÃO EM ESPORTES OU COMPETIÇÕES. Elas relacionam suas habilidades em participar destas atividades e como estas são influenciadas pelo seu problema do joelho. Considere os últimos três meses para responder estas questões.

9) Quanto de limitação você apresenta para movimentos rotacionais ou de mudanças bruscas de direção?

$\begin{array}{cc}0 & 100 \\ \text { Totalmente } & \text { Sem } \\ \text { limitado } & \text { limitações }\end{array}$

10) Quanto você se preocupa com o fato de que atividades esportivas ou recreacionais possam piorar o estado do seu joelho?

0 100

Muito

Sem

preocupado

preocupações

11) Qual é o seu nível atual de performance nas atividades recreacionais ou esportivas, comparando com a performance antes da lesão?

0 100

Totalmente Sem

limitado limitações

12) Considerando as atividades ou esportes que você se envolve hoje, quanto as suas expectativas mudaram devido ao estado do seu joelho?

0 100

Diminuíram
completamente Não diminuíram nada

13) Você tem que praticar suas atividades esportivas ou recreacionais com cuidado? (Marque o "I" no início da linha à esquerda se você se encontra incapaz de praticar atividades esportivas ou recreacionais devido ao seu joelho)

0

Sempre pratico

com cuidado
100

Nunca pratico

com cuidado

14) Quanto de medo você tem de sua rótula (patela) sair do lugar durante atividades esportivas ou recreacionais?

0 100

\section{Muito medo}

Sem medo

Apêndice 1 (continued). 
15) Você se preocupa com condições do ambiente, como um campo ou solo molhado, ou ainda uma quadra rápida, ou então com o tipo do piso do ginásio, quando está envolvido com suas atividades esportivas ou recreacionais? (Marque o "/" no início da linha à esquerda se você se encontra incapaz de praticar atividades esportivas ou recreacionais devido ao seu joelho)

$\begin{array}{cl}\text { Muito } & 100 \\ \text { preocupado } & \text { Nada } \\ \text { preocupado }\end{array}$

16) Você se frustra quando pensa no seu joelho ao considerar a realização de atividades recreacionais ou esportivas?

0 100

\section{Extremamente frustrado} Não me sinto frustrado

17) Qual é o grau de dificuldade para você "dar o máximo de si" nas atividades esportivas ou recreacionais? (Marque o "/" no início da linha à esquerda se você se encontra incapaz de praticar atividades esportivas ou recreacionais devido ao seu joelho)

0 100

Extremamente Não sinto difícil dificuldade

18) Você sente medo ao praticar esportes de contato? (circule o item "N. S. A." - Não Se Aplica - à direita da linha, caso o motivo pelo qual você não pratica esportes de contato não seja o seu joelho)

$\begin{array}{cc}\text { Muito } & 100 \\ \text { medo } & \text { Sem } \\ \text { medo }\end{array}$

As questões abaixo se relacionam aos dois principais esportes ou atividades recreacionais que você realiza. Por favor escreva estas duas atividades em ordem de importância.

1.

2.

19) Quanto de limitação você encontra quando pratica o esporte ou atividade recreacional listado como número 1? (Posicione o "/" no início da linha à esquerda se você se encontra incapaz de praticar esportes ou atividades recreacionais devido ao seu joelho)

$\begin{array}{ll}0 & 100 \\ \text { Extremamente } & \text { Sem } \\ \text { limitado } & \text { limitações }\end{array}$

20) Quanto de limitação você encontra quando pratica o esporte ou atividade recreacional listado como número 2? (Posicione o "/" no início da linha à esquerda se você se encontra incapaz de praticar esportes ou atividades recreacionais devido ao seu joelho)

$$
0
$$
100

\section{Extremamente}

Sem

limitado

limitações

Apêndice 1 (continued). 


\section{SEÇÃO D: ESTILO DE VIDA}

As questões abaixo lidam com seu estilo de vida em geral e devem ser consideradas fora do ambiente de trabalho e de esportes ou atividades recreacionais. São relacionadas ao seu joelho no que diz respeito a instabilidade patelofemoral (rótula que sai do lugar).

21) Você se preocupa com problemas de segurança em geral (como ao carregar uma criança pequena, jardinagem...) no que diz respeito ao seu joelho com instabilidade patelofemoral?

$$
0
$$
100

Extremamente

Sem

preocupado

preocupações

22) Quanto da sua habilidade de se exercitar e se manter em forma esteve limitada devido ao seu problema no joelho?

$$
\begin{array}{ll}
\mathrm{O} & 100 \\
\text { Completamente } & \text { Sem } \\
\text { limitado } & \text { limitações }
\end{array}
$$

23) Quanto do seu prazer na sua vida foi limitado pelo seu problema no joelho?

$$
0
$$
100

\section{Completamente}

Sem

limitado

limitações

24) Com que frequência você se dá conta do seu problema no joelho?

$$
0
$$
100

O tempo

Nunca

todo

25) Considerando o seu estilo de vida, quanto você se preocupa com o seu joelho em relação às atividades que você e sua família realizam?

$$
0
$$
100

Extremamente

Não me

preocupado

preocupo

26) Você teve que modificar seu estilo de vida para evitar atividades que pudessem machucar o seu joelho?

$$
\begin{array}{cc}
\text { Modifiquei } & 100 \\
\text { completamente } & \text { Não } \\
\text { modifiquei }
\end{array}
$$

Apêndice 1 (continued). 


\section{SEÇÃO E: SOCIAL E EMOCIONAL}

As questões abaixo consideram suas atitudes e sentimentos e a maneira que eles se relacionam com seu joelho com instabilidade patelofemoral (rótula que sai do lugar). Considere os últimos três meses.

27) Quanto você se incomoda com o fato de não ser mais competitivo por conta do seu problema no joelho? (Marque o "/" ao final da linha à direita, para além do 100 se suas necessidades competitivas estão sendo alcançadas. Marque o "/" ao início da linha, sobre o 0 se você não tiver necessidades competitivas)

0 100

Extremamente

Nada

incomodado

incomodado

28) Você apresentou alguma dificuldade para superar psicologicamente os problemas relacionados ao seu joelho?

0 100

Dificuldade Sem qualquer extrema dificuldade

29) Com que frequência você se sente apreensivo ou com medo em relação ao seu joelho?

0 100

O tempo Em nenhum

todo momento

30) Quanto você se incomoda por uma falta de confiança no seu joelho?

0 100

\section{Extremamente}

incomodado
Sem nenhum

incômodo

31) Se você pudesse quantificar, quanto de medo você tem de desenvolver uma nova lesão no mesmo joelho envolvido?

0 100

Muito medo Sem medo

algum

Obrigado por completar este questionário.

Apêndice 1 (continued). 